University of Wollongong

Research Online

Faculty of Engineering and Information

Faculty of Engineering and Information

Sciences - Papers: Part A

Sciences

$1-1-2014$

The pricing of credit default swaps under a generalized mixed fractional

Brownian motion

Xinjiang He

University of Wollongong, xh016@uowmail.edu.au

Wenting Chen

University of Wollongong,wtchen@uow.edu.au

Follow this and additional works at: https://ro.uow.edu.au/eispapers

Part of the Engineering Commons, and the Science and Technology Studies Commons

Research Online is the open access institutional repository for the University of Wollongong. For further information contact the UOW Library: research-pubs@uow.edu.au 


\title{
The pricing of credit default swaps under a generalized mixed fractional Brownian motion
}

\begin{abstract}
In this paper, we consider the pricing of the CDS (credit default swap) under a GMFBM (generalized mixed fractional Brownian motion) model. As the name suggests, the GMFBM model is indeed a generalization of all the FBM (fractional Brownian motion) models used in the literature, and is proved to be able to effectively capture the long-range dependence of the stock returns. To develop the pricing mechanics of the CDS, we firstly derive a sufficient condition for the market modeled under the GMFBM to be arbitrage free. Then under the risk-neutral assumption, the CDS is fairly priced by investigating the two legs of the cash flow involved. The price we obtained involves elementary functions only, and can be easily implemented for practical purpose. Finally, based on numerical experiments, we analyze quantitatively the impacts of different parameters on the prices of the CDS. Interestingly, in comparison with all the other FBM models documented in the literature, the results produced from the GMFBM model are in a better agreement with those calculated from the classical Black-Scholes model. 2014 Published by Elsevier B.V. All rights reserved.
\end{abstract}

\section{Keywords}

pricing, default, credit, swaps, under, generalized, mixed, fractional, brownian, motion

\section{Disciplines}

Engineering | Science and Technology Studies

\section{Publication Details}

He, X. \& Chen, W. (2014). The pricing of credit default swaps under a generalized mixed fractional Brownian motion. Physica A: Statistical Mechanics and its Applications, 404 (June), 26-33. 


\title{
The pricing of credit default swaps under a generalized mixed fractional Brownian motion
}

\author{
Xinjiang $\mathrm{HE}^{\mathrm{a}}$, Wenting CHEN ${ }^{\mathrm{b}}$ \\ ${ }^{a}$ Department of Mathematics, Donghua University, Shanghai, China \\ ${ }^{b}$ Corresponding author. School of Mathematics and Applied Statistics, University of Wollongong NSW 2522, Australia.
}

\begin{abstract}
In this paper, we consider the pricing of the CDS (credit default swap) under a GMFBM (generalized mixed fractional Brownian motion) model. As the name suggests, the GMFBM model is indeed a generalization of all the FBM (fractional Brownian motion) models used in the literature, and is proved to be able to effectively capture the long-range dependence of the stock returns. To develop the pricing mechanics of the CDS, we firstly derive a sufficient condition for the market modeled under the GMFBM to be arbitrage free. Then under the risk-neutral assumption, the CDS is fairly priced by investigating the two legs of the cash flow involved. The price we obtained involves elementary functions only, and can be easily implemented for practical purpose. Finally, based on numerical experiments, we analyze quantitatively the impacts of different parameters on the prices of the CDS. Interestingly, in comparison with all the other FBM models documented in the literature, the results produced from the GMFBM model are in a better agreement with those calculated from the classical Black-Scholes model.
\end{abstract}

\section{(c) 2013 Published by Elsevier Ltd.}

Keywords: Generalized mixed fractional Brownian motion, Credit default swaps, Pricing swaps.

\section{1. introduction}

Credit risk is the risk brought by the party which is unable to perform or meet its settled obligations because of bankruptcy or other serious financial problems during the transaction. Such a risk has long been considered as one of the most adverse factors for the development of the financial market. The management and control of credit risk have thus become again one of the main topics in quantitative finance research after the 2008 Global Finance Crisis took place.

One of the earliest and most basic credit derivatives to effectively manage the credit risk is the so-called CDS (credit default swap). A CDS is a contract transferring credit risk of the assets from its buyer to the seller. The buyer buys the contract from the seller by paying fixed amount of fees regularly as specified in the contract, whereas the seller who is willing to undertake the credit risk has the obligation to compensate the buyer in case of default. The credit risk becomes tradable and manageable after the use of the CDS. With the growing demand for credit derivatives, the accurate determination of the price of the CDS becomes essentially important, because the CDS is usually regarded as the basis of many other credit derivatives.

In the literature, the theoretical pricing of credit derivatives, including the CDS, has received plenty of attentions. For example, Longstaff and Schwartz [14] solved the pricing of credit spread options under an exogenous process. de 
Malherbe [6] has used a probabilistic approach to determine the price of the CDS under the Poisson process. With stochastic intensity models adopted for the default events, Brigo \& Chourdakis [3] considered counterparty risk for CDS in presence of correlation between default of the counterparty and default of the CDS reference credit. It should be pointed out that the accurate determination of a CDS price mainly depends upon the chosen model for the credit risk. In this area, two kinds of models are widely used: the reduced form models and the structural models. The former models originate in [12], and are further developed in [7, 15, 24]. Under such models, the authors assume that the likelihood and intensity of the default can be derived from the historic data of the company. This assumption, however, ignores the impact of interactions of market risks and other information. The structural models, as another alternative, use the evolution of the structure variable of the asset of the company and the value of the debt to determine the time when the default occurs. In this category, Merton [19] is the one who first used the structural model to characterize the breach by assuming that the default would occur if the company is insolvent. His model was then further development in [23] by taking the stochastic interest rate into account.

Although appealing, the Merton model fails to capture the long-range dependence of real asset returns, as suggested in many recent empirical studies [11, 18, 22, 31]. It should be remarked that the idea that the asset returns could exhibit long-range dependence was suggested in [16], and subsequently observed in many empirical studies (see [17] and the references therein). To incorporate this issue, the so-called FBM (fractional Brownian motion) is introduced. In addition to the ability of capturing the long-range dependence of the asset returns, this process can also produce a burstiness in its sample path, which agrees well with another important behavior of the time series of the asset returns. It should be pointed out that the FBM is neither a Markov nor a semi-martingale, which ceased the application of classical Itô theory [13]. To tackle this problem, the Wick product is introduced to define the stochastic integration, and the FBM is wickbitrage free [2]. However, it should be further remarked that the concept of wickbitrage does not correspond to arbitrage in the intuitive sense. A wickbitrage-free model such as the FMB may in some cases still lead to implementable naive arbitrage opportunities, as pointed in $[2,5]$. To resolve this issue while taking into account the long memory property, the MFBM (mixed fractional Brownian motion) is introduced, which is a linear combination of a BM and a FBM. For a detailed survey on the pricing of various derivatives under the MFBM, we refer to $[25,26,29]$ and the references therein.

Recently, the MFBM has been further generalized by Thäle [28] to the GMFBM (generalized mixed fractional Brownian motion), which is a linear combination of a countable number of BMs and FBMs. This model includes the BM, FBM, and MFBM as its simplest cases, and is adopted for the current work. Under this model, we have shown, in Section 2, that the market under the GMFBM could be arbitrage free under certain parameter settings. The pricing of the CDS is then developed under the risk-neutral assumption. The solution we have found is written in terms of elementary functions, and is very easy to implement for practical purpose. It should be further remarked that although some preliminary work regarding the pricing of the CDS has been done in [10], it is not trivial to extend the methodology to the current GMFBM model. With the availability of the current solution, the CDS prices under different FBM models can be obtained straightforwardly because of the generality of the GMFBM model. Furthermore, through numerical experiments, we discuss quantitatively the impacts of different parameters on the prices of the CDS.

The paper is organized as follows. In Section 2, we briefly review the GMFBM model, and give a sufficient condition for the GMFBM model to be a martingale. In Section 3, we derive a closed-form analytical solution for the price of the CDS. In Section 4, numerical examples and some useful discussions are presented. Concluding remarks are given in the last section.

\section{Generalized mixed fractional Brownian motion}

Before considering the pricing of the CDS, we shall briefly review some background concerning the GMFBM. For a more detailed treatment, we refer to $[28,27]$. 
The concept of a GMFBM is recently introduced by Thäle [28] based upon the well known literature of FBM and its generalization MFBM. It is formally defined as follows.

Definition 2.1. A GMFBM of parameter $H=\left(H_{1}, \cdots, H_{N}\right)$ and $\alpha=\left(\alpha_{1}, \cdots, \alpha_{N}\right)$ is a stochastic process $Z^{H}=$ $\left(Z_{t}^{H}\right)_{t \geq 0}=\left(Z_{t}^{H, \alpha}\right)_{t \geq 0}$ defined on some probability space $(\Omega, F, P)$ by

$$
Z^{H, \alpha}=\sum_{k=1}^{N} \alpha_{k} B_{H_{k}}(t)
$$

where $\left(B_{H_{k}}(t)\right)_{t \geq 0}$ are independent FBMs of Hurst parameters $H_{k}$, for $k=1, \cdots, N$.

According to this definition, it is clear that the GMFBM model is a generalization of all the FBM models considered in the literature. Such a generalized model degenerates to the single FBM model with $N=1$, the BM model with $N=1$ and $H_{1}=\frac{1}{2}$, and the MFBM model with $N=2$ and $H_{1}=\frac{1}{2}$. As shown in [27], the GMFBM also has the following properties.

\section{Proposition 1. The GMFBM satisfies}

(i) $Z^{H}=\left(Z_{t}^{H, \alpha}\right)_{t \geq 0}$ is a centered Gaussian process with variance $\sum_{k=1}^{N} \alpha_{k}^{2} t^{2 H_{k}}$ and covariance $E\left(Z_{t}^{H, \alpha} Z_{s}^{H, \alpha}\right)=$ $\frac{1}{2} \sum_{k=1}^{N} \alpha_{k}^{2}\left(t^{2 H_{k}}+s^{2 H_{k}}\right)-|t-s|^{2 H_{k}}$.

(ii) The increments of $Z^{H}$ are stationary and $s_{\left(c_{1}, \cdots, c_{N} ; H_{1}, \cdots, c_{N}\right)}$ self similar in the sense that $\sum_{k=1}^{N} \alpha_{k} B_{H_{k}}(t)=\sum_{k=1}^{N} \alpha_{k} c_{k}^{-H_{k}} B_{H_{k}}\left(c_{k} t\right)$ in distribution.

(iii) $Z^{H}$ exhibits a long-range dependence if and only if there exists $k$ with $H_{k}>\frac{1}{2}$.

(iv) Every simple path of $Z^{H}$ is almost surely nowhere differentiable.

It should be remarked that the classical Ito theory could not be applied to define a stochastic integral over the GMFBM, because it is neither a Markov nor a semi-martingale unless under certain parameter settings [8]. Duncan et al. [8] suggested that the Wick product be used in the definition of the stochastic integral. Once this special product is adopted, the stochastic integral defined will have a zero-mean property, which is very useful for both theoretic development as well as practical application. With the use of the Wick product, a new concept of wickbitrage is defined [9]. It is also suggested in [9] that the FBM models are "free of arbitrage" in the sense that they are free of wickbitrage.

While mathematically appealing, the introduction of the Wick product can, however, not prevent arbitrage in the regular sense. Recently, some arbitrage opportunities have already been found under the so-called "wickbitrage free" models $[2,5]$. The main reason why the use of the Wick product fails to prevent arbitrage is that with the Itô integral replaced by the integral defined on Wick product, the definitions of portfolio value and self-financing portfolios, two concepts usually used to determine whether the market considered is arbitrage free or not, are totally different from their standard counterparts, as pointed out in [2]. For the MFBM model, it is shown in [4] that with certain constraints placed on the Hurst parameter, the MFBM could be equivalent to multiples of a standard BM, and thus the corresponding market is arbitrage free in the regular sense. In the following, we shall extend such a result to the GMFBM model based on the recent work did in [30]. It should be remarked that the equivalence theorem established in [30] is much more general than the one derived in [4], because the former considers a linear combinational of a finite number of FBMs, whereas the latter concentrates on the sum of a BM and a FBM only.

Without loss of generality, we consider a GMFBM $Z^{H}$ with $H_{1}<H_{2}<\cdots<H_{N}$, and $\alpha_{1}, \cdots, \alpha_{N} \in \mathbb{R} \backslash\{0\}$. From (i) of Proposition 1 and [30], it is known that $Z^{H}$ is a Gaussian si-process with spectral density $f(\lambda)=\sum_{k=1}^{N} \alpha_{k}^{2} f_{H_{k}}(\lambda)$, 
where $f_{H_{k}}(\lambda)$ is the spectral density of a FBM with Hurst parameter $H_{k} \in(0,1)$, and is defined as

$$
f_{H_{k}}(\lambda)=D_{H_{k}}|\lambda|^{1-2 H_{k}}, \quad D_{H_{k}}=\frac{\sin \left(\pi H_{k}\right) \Gamma\left(1+2 H_{k}\right)}{2 \pi} .
$$

According to the fact that the Hurst parameters in $Z^{H}$ are increasing with the subscript, it is not difficult to show that $f$ admits the following asymptotic behavior at $\lambda=\infty$ :

$$
f(\lambda)=\alpha_{1}^{2} f_{H_{1}}+\alpha_{2}^{2} D_{H_{2}}|\lambda|^{1-2 H_{2}}(1+O(1)) .
$$

Intuitively, from (2.1), it is reasonable to write $Z^{H}$ as the sum of a multiple of an FBM with Hurst parameter $H_{1}$ and a second, independent Gauss si-process whose spectral density behaves like a multiple of $|\lambda|^{1-2 \mathrm{H}_{2}}$ near infinity, as already pointed in [30]. Indeed, van Zanten [30] has shown that if $H_{2}-H_{1}>\frac{1}{4}, Z^{H}$ is locally equivalent to $\alpha_{1} B_{H_{1}}(t)$. Here, "Locally equivalent" refers to the equivalence on $[0, T]$ for every $T>0$. We shall briefly outline the proof of the equivalence in the following theorem.

Theorem 1. Let $Z^{H}$ be a GMFBM with Hurst parameters $H_{1}<H_{2}<\cdots<H_{N}$, and $\alpha_{1}, \cdots, \alpha_{N} \in \mathbb{R} \backslash\{0\}$. If $H_{2}-H_{1}>\frac{1}{4}$, then $Z^{H}$ and $Y=\alpha_{1} B_{t}^{H_{1}}$ are locally equivalent.

Proof. Let $f_{0}(\lambda)$ and $f_{1}(\lambda)$ be the spectral density of $Y$ and $Z^{H}$, respectively. According to [30], the main step of showing the equivalence of the two Gauss si-processes is to check the integrability of

$$
\int_{c}^{\infty}\left[\frac{f_{1}(\lambda)-f_{0}(\lambda)}{f_{0}(\lambda)}\right]^{2} S_{T}^{0}(\lambda, \lambda) f_{0}(\lambda) d \lambda
$$

where $S_{T}^{0}$ is the reproducing kernel of $Y$. According to the asymptotic behavior of $S_{T}^{0}$ obtained in [30] together with the explicit expressions of $f_{0}(\lambda)$ and $f_{1}(\lambda)$, it is not difficult to show that there exists a constant $M$, such that

$$
\text { । }\left.\left[\frac{f_{1}(\lambda)-f_{0}(\lambda)}{f_{0}(\lambda)}\right]^{2} S_{T}^{0}(\lambda, \lambda) f_{0}(\lambda)|\leq M| \lambda\right|^{4\left(H_{1}-H_{2}\right)} .
$$

The integrability of (2.2) for the case when $H_{2}-H_{1}>\frac{1}{4}$ can then be obtained by the Cauchy approach. This has completed the proof

From Theorem 1, one can straightforwardly obtain the following corollary.

Corollary 2.1. In a GMFBM $\left(Z_{t}^{H, \alpha}\right)_{t \geq 0}$ market with Hurst parameters $H=\left(H_{1}, \cdots, H_{N}\right)$ satisfying $H_{1}<H_{2}<\cdots<$ $H_{N}$ and $\alpha=\left(\alpha_{1}, \cdots, \alpha_{N}\right) \in \mathbb{R} \backslash\{0\}$, no arbitrage opportunities exist if

$$
H_{1}=\frac{1}{2}, \frac{3}{4}<H_{2}<H_{3}<\cdots<H_{N}<1 .
$$

Proof. According to Theorem 1, $\left(Z_{t}^{H, \alpha}\right)_{t \geq 0}$ is equivalent to $\alpha_{1} B_{t}^{\frac{1}{2}}$, where $B_{t}^{\frac{1}{2}}$ is the standard BM. Since the market under the standard BM is both complete and arbitrage free [5], the result of this corollary can be obtained straightforwardly.

It is not difficult to show that when $N=2$ and $\alpha_{2}=1$, the GMFBM considered in Corollary 2.1 degenerates to the MFBM considered in [4], and our result described in Corollary 2.1 becomes exactly the equivalence theorem established in [4] as well.

\section{Closed-form analytical solution}

In this section, we shall derive closed-form analytical expression for the price of the CDS under the CMFBM model. We shall firstly calculate the default probability of the company, based on which, the CDS price can then be obtained by examining its cash flows. 


\subsection{Default probability of the company}

One of the key steps of deriving the price of the CDS is to determine the default probability of the company. As the name suggests, the so-called default probability describes the likelihood of a default of a financial institution over a particular time horizon. In this subsection, we shall calculate this important quantity, which will pave the way to derive the price of the CDS.

We now consider a GMFBM market consisting of a bank account and a risky asset of the firm (i.e., reference asset). The value $P(t)$ of one dollar in the bank account at time $t$ satisfies

$$
d P(t)=r P(t) d t, \quad P(0)=1,
$$

where the constant $r>0$ denotes the risk-free interest rate. Furthermore, the value of the reference asset $V(t)$ follows the GMFBM under the probability measure $\mathbb{P}$ :

$$
d V_{t}=(\mu-\rho) V_{t} d t+\sigma V_{t} \diamond d \bar{X}_{t} .
$$

Here, $\diamond$ denotes the Wick product, $\mu$ is the expected rate of asset return, $\sigma$ is the volatility, $\rho$ is the dividend yield of the underlying, and $\bar{X}_{t}$ is the GMFBM expressed as $\bar{X}_{t}=\sum_{i=1}^{N} \alpha_{i} \bar{B}_{H_{i}}(t)$, with $\left(\bar{B}_{H_{i}}(t)\right)_{t \geq 0}$ being independent FBMs of Hurst parameters $H_{i}, i=1,2 \cdots N$.

According to the fractional Girsanov theorem [13], it is known that under the risk-neutral measure $\mathbb{Q}, V_{t}$ should satisfy

$$
d V_{t}=(r-\rho) V_{t} d t+\sigma V_{t} \diamond d X_{t},
$$

where $X_{t}=\frac{\mu-r}{\sigma} t+\bar{X}_{t}$. Now, using Wick calculus, the solution of (3.4) can be found as

$$
\begin{aligned}
V_{t} & =V_{0} \diamond \exp \diamond\left[(r-\rho) t+\sum_{i=1}^{N} \int_{0}^{t} \sigma \alpha_{i} d B_{H_{i}}(t)\right], \\
& =V_{t} e^{(r-\rho) t} \exp \diamond\left[\sum_{i=1}^{N} \int_{0}^{t} \sigma \alpha_{i} d B_{H_{i}}(t)\right] .
\end{aligned}
$$

According to the communicative and associative law of Wick exponentials [1], (3.5) can be further simplified as

$$
V_{t}=V_{0} \exp \left[(r-\rho) t+\sum_{i=1}^{N} \int_{0}^{t} \sigma \alpha_{i} d B_{H_{i}}(t)-\frac{1}{2} \sum_{i=1}^{N} \int_{0}^{t}\left(M_{H_{i}} \sigma \alpha_{i}\right)^{2} d s\right]
$$

where the operator $M_{H_{i}}$ is defined as

$$
M_{H_{i}} f(x)=-\frac{d}{d x} \frac{C_{H_{i}}}{\left(H_{i}-\frac{1}{2}\right)} \int_{R}(t-x)|t-x|^{H_{i}-\frac{3}{2}} f(t) d t,
$$

with $f \in S(R)$ and $C_{H_{i}}=\left\{2 \Gamma\left(H_{i}-\frac{1}{2}\right) \cos \left[\frac{\pi}{2}\left(H_{i}-\frac{1}{2}\right)\right]\right\}^{-1}\left[\Gamma\left(2 H_{i}+1\right) \sin \left(\pi H_{i}\right)\right]^{\frac{1}{2}}$.

Let $M_{H_{i}}[0, t]=M I_{[0, t](x)}$, where $I_{[a, b]}$ is the indicator function of the interval $[0, t]$, and consequently, (3.6) can be rewritten as

$$
\text { (3.6) }=V_{0} \exp \left[(r-\rho) t+\sum_{i=1}^{N} \int_{0}^{t} \sigma \alpha_{i} d B_{H_{i}}(t)-\frac{1}{2} \sum_{i=1}^{N} \int_{R}\left(M_{H_{i}}[0, t] \sigma \alpha_{i}\right)^{2} d s\right] .
$$

According to the Parceval Theorem [1], it is known that (3.8) can be further simplified as

$$
(3.8)=V_{0} \exp \left[(r-\rho) t+\sum_{i=1}^{N} \sigma \alpha_{i} B_{H_{i}}(t)-\frac{1}{2} \sum_{i=1}^{N} \frac{\sigma^{2} \alpha_{i}^{2}}{2 \pi} \int_{R} \widehat{\left.\left.\left(\widehat{M_{H_{i}}[0, t}\right](\xi)\right)^{2} d \xi\right]},\right.
$$




$$
\begin{aligned}
& =V_{0} \exp \left[(r-\rho) t+\sum_{i=1}^{N} \sigma \alpha_{i} B_{H_{i}}(t)-\frac{1}{2} \sum_{i=1}^{N} \frac{\sigma^{2} \alpha_{i}^{2}}{2 \pi} \int_{R}|\xi|^{1-2 H_{i}} \frac{\left|e^{-i t \xi}-1\right|}{|\xi|^{2}} d \xi\right] \\
& =V_{0} \exp \left[(r-\rho) t+\sum_{i=1}^{N} \sigma \alpha_{i} B_{H_{i}}(t)-\frac{1}{2} \sum_{i=1}^{N} \sigma^{2} \alpha_{i}^{2} t^{2 H_{i}}\right]
\end{aligned}
$$

Now, let $D$ denote the face value of the loan. If the asset value of the company is less than or equal to $D$ at $T$, the default of the company is triggered. Therefore, the default probability of the company is equivalent to the probability that $V_{T} \leq D$, i.e., $P\left(V_{T} \leq D\right)$. According to (i) of Proposition 1, the default probability of the company can be calculated as

$$
\begin{aligned}
P\left(V_{T} \leq D\right) & =P\left(V_{0} \exp \left[(r-\rho) T+\sum_{i=1}^{N} \sigma \alpha_{i} B_{H_{i}}(T)-\frac{1}{2} \sum_{i=1}^{N} \sigma^{2} \alpha_{i}^{2} T^{2 H_{i}}\right] \leq D\right), \\
& =P\left(\sum_{i=1}^{N} \sigma \alpha_{i} B_{H_{i}}(T) \leq \ln \frac{D}{V_{0}}-(r-\rho) T+\frac{1}{2} \sum_{i=1}^{N} \sigma^{2} \alpha_{i}^{2} T^{2 H_{i}}\right), \\
& =P\left(\frac{\sum_{i=1}^{N} \sigma \alpha_{i} B_{H_{i}}(T)}{\sqrt{\sum_{i=1}^{N} \sigma^{2} \alpha_{i}^{2} T^{2 H_{i}}}} \leq \frac{\ln \frac{D}{V_{0}}-(r-\rho) T+\frac{1}{2} \sum_{i=1}^{N} \sigma^{2} \alpha_{i}^{2} T^{2 H_{i}}}{\sqrt{\sum_{i=1}^{N} \sigma^{2} \alpha_{i}^{2} T^{2 H_{i}}}}\right), \\
& =N(d),
\end{aligned}
$$

where $\frac{D}{V_{0}}$ is the initial leverage of the target company, $N(\cdot)$ is the standard normal distribution, and $d$ is defined as

$$
d=\frac{\ln \frac{D}{V_{0}}-(r-\rho) T+\frac{1}{2} \sum_{i=1}^{N} \sigma^{2} \alpha_{i}^{2} T^{2 H_{i}}}{\sqrt{\sum_{i=1}^{N} \sigma^{2} \alpha_{i}^{2} T^{2 H_{i}}}}
$$

With the default probability of the company available, it suffices for us to determine the price for the CDS. This will be illustrated in detail in the next subsection.

\subsection{Valuation of the credit default swaps}

A CDS is a financial agreement that the seller will compensate the buyer in the event of a loan default or other credit event [20]. The buyer of the CDS should make a series of payments to the seller and, in exchange, will receive a payoff if the loan defaults. It should be pointed out that in swap terminology, the price of a particular swap differs from its value. In terms of the CDS considered, which is a special swap, its price refers to the regular fixed fee that the buyer pays to the seller, and is usually measured by a percentage of the reference asset. A schematic diagram of the transaction mechanics of the CDS is shown in Fig 1.

To determine the price $c$ of the CDS, we need to further set up the cash flow of a CDS contract. Suppose that the buyer of the CDS makes fixed amount payments $c M$ to the seller at discrete times $t_{i}, i=1,2, \cdots, n$, where $0=t_{1}<t_{2}<\cdots<t_{n}=T, T$ is the expiration date, and $M$ is the nominal value of the reference asset. We further assume that the seller has the obligation to compensate the loss of the buyer only at $T$, by paying $(1-L) M$ to the buyer if the company defaults with $L$ being the satisfaction rate.

Depicted in Fig 2 is the cash flow of the CDS. From this figure, it is clear that there are two parts of cash flows involved in a certain CDS contract. One is the fixed amount of cash the buyer pays to the seller at each discrete time $t_{i}, i=1,2, \cdots, n$. Assuming that the bank interest rate is of continuous compounding, the present value of this cash flow is $P_{1}=\sum_{i=1}^{n} c M e^{-r t_{i}}$. The other part of the cash flow will come into existence only if the default of the company is triggered. According to the CDS contract, it is known that the buyer could ask for $(1-L) M$ amount of compensation from the seller. Therefore, the present value of this part is $P_{2}=e^{-r T} M(1-L) P\left(V_{T} \leq D\right)$. 


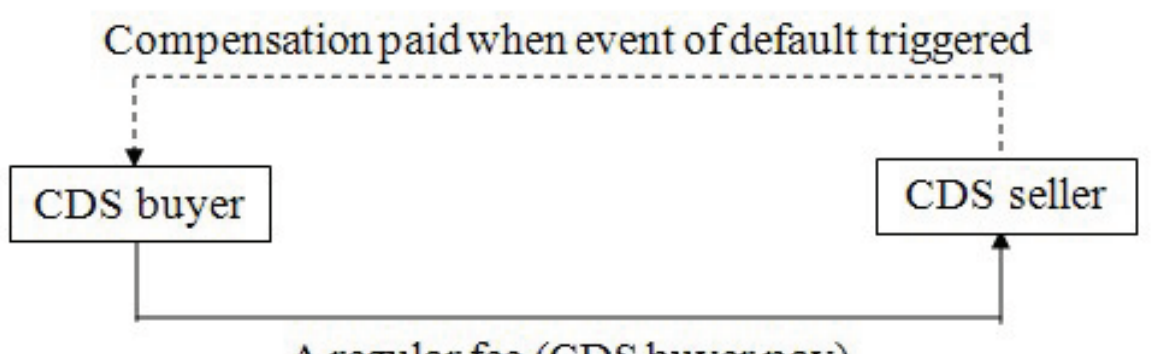

A regular fee (CDS buyer pay)

Figure 1. The transaction mechanics of the CDS

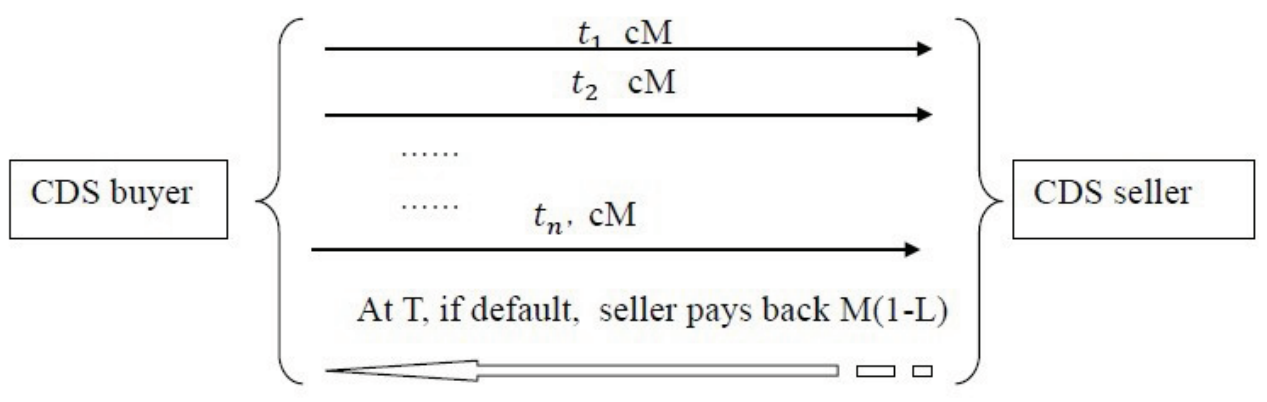

Figure 2. Cash flow of the CDS 
Similarly to a forward/futures contract, when a CDS is initiated, it should be fair to both parties. This implies that the cash flow of the CDS should be zero when the contract is entered into. We obtain $P_{1}=P_{2}$, from which, the price of the CDS can be solved as

$$
c=\frac{e^{-r T}(1-L) N(d)}{\sum_{i=1}^{n} e^{-r t_{i}}},
$$

where $d$ is defined in (3.11). We remark that the CDS price obtained here can easily degenerate to the corresponding prices under other FBM models, because of the generality of the GMFBM model, as already pointed out in Section 2.

With the price of the CDS available, one could further determine the value of this contract by examining the cash flow at the present time $t$. We let $f(t)$ be the cash flow the CDS at any time $t(0 \leq t \leq T)$, i.e.,

$$
f(t)=\left|\sum_{i=1}^{n} c M e^{-r\left(t_{i}-t\right)}-M(1-L) N(d) e^{-r(T-t)}\right| .
$$

By substituting (3.12) into the above expression of $f(t)$, it is not difficult to show that $f(t)=0$. In other words, the net present value of a CDS is always zero. However, if the market interest rate is changing all the time, the CDS could take on positive or negative value, depending on the passage of time.

\section{Numerical examples and discussions}

In this section, we shall present some numerical results and carry out some discussions on the newly derived formula. In particular, some quantitative analyses on the impacts of different parameters will be conducted.

Depicted in Fig 3(a) are three sets of default probabilities calculated from the BM, MFBM, and GMFBM, respectively. The absolute differences among the three models are further shown in Fig 3(b). For comparison purpose, the coefficients $\alpha_{i}$ s are chosen such that both the MFBM and the GMFBM have the same quartiles as the BS distribution with volatility $\sigma$, while all the other parameters are kept the same. In particular, we set $\alpha_{i}=\frac{T^{1-H_{i}}}{\sqrt{N}}$ for $i=1, \cdots, N$, where $T$ is the maturity date, $H_{i}=0.5+\frac{0.95-0.5}{N-1}(i-1)$ is the Hurst parameter associated with $B_{H_{i}}(t)$, and $N$ is the total number of FBMs involved in the GMFBM. According to Corollary 2.1, it can be deduced that under the above parameter settings, the market under the GMFBM is arbitrage free. From Fig 3, it is interesting to notice that the default probability calculated from the GMFBM model is much closer to the one under the BS model than that of the MFBM.

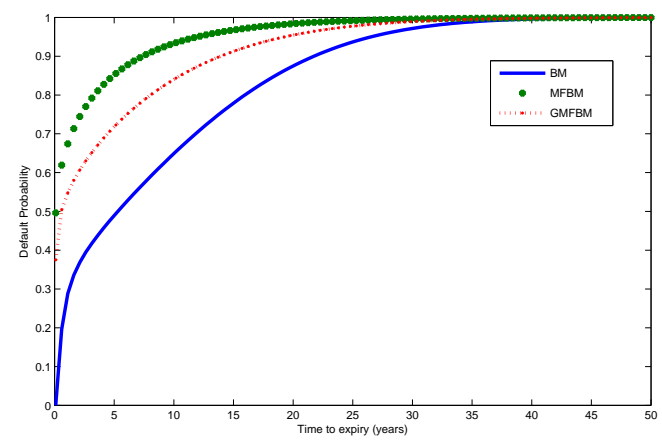

(a) Default probability with $D=80, T=50$.

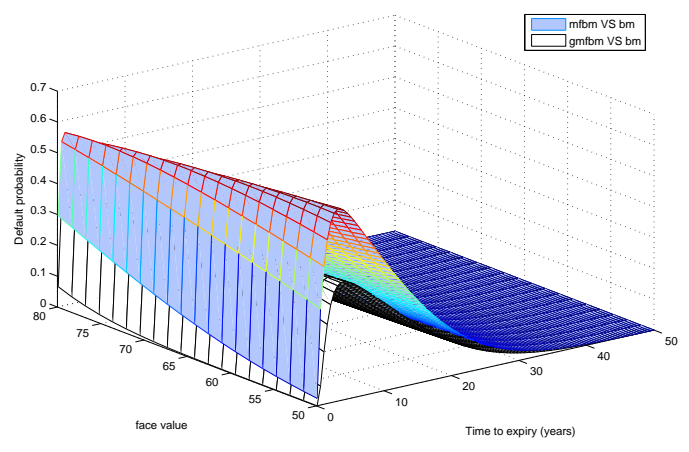

(b) Absolute differences for $T=50, D \in(50,80)$.

Figure 3. Comparison of default probabilities under different models. Model parameters are $V_{0}=100, r=0.06, \sigma=0.15, \rho=0.01, N=50$ for GMFBM, and $N=2$ for MFBM. 
After examining the default probability, we shall turn to investigate the quantitative impacts of different parameters on the price of the CDS. We shall concentrate on analyzing the effects of the expiration time $T$, the number of discrete payments $n$, the number of FBMs involved in the GMFBM, because the impacts of the rest of parameters can be observed straightforwardly from the closed-form analytical expression of $c$.

Depicted in Fig 4(a) are the CDS prices under three different models as a function of the expiration time $T$. From this figure, one can clearly observe that the price increases when $T$ becomes larger. However, after a certain value of $T$ and onwards, the price decreases as $T$ increases. This is indeed reasonable. For a reasonably small $T$, the CDS price is dominated by the default probability, which is monotonically increasing with $T$, as shown in Fig 3(a). However, when $T$ becomes very large, the dominant factor becomes $\frac{e^{-r T}}{\sum_{i=1}^{n} e^{-r t_{i}}}$, because the default probability is approaching 1 for quite large $T$ values. In this case, the price would drop if $T$ further increases, as the dominant factor is a monotonic decreasing function with $T$ if $n$ is kept constant, which is the assumption here. On the other hand, for any fixed expiration time $T$, the price of the CDS varies inversely with respect to the number of the payments, as shown in Fig 4(b). Financially, this makes sense, too, because if the buyer of the CDS pays to the seller more often, he needs to pay less each time, provided that the total amount of money the buyer could receive in case of default remains unchanged.

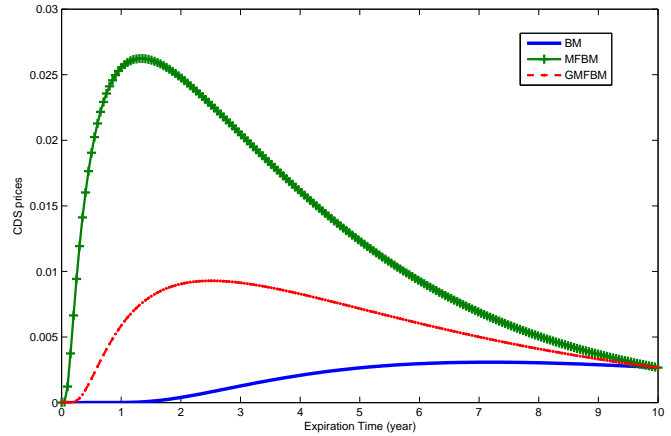

(a) Different $T$

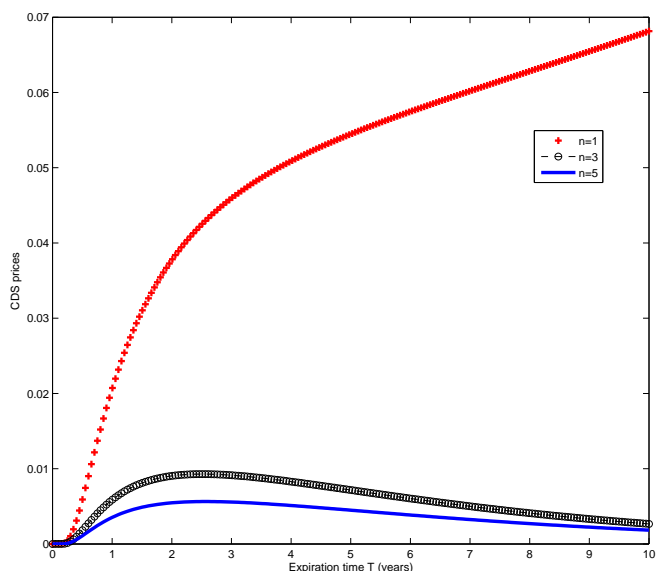

(b) Different no. of discrete payments

Figure 4. Variations of CDS prices with $T$ and $n$. Model parameters are $V_{0}=100, r=0.06, \sigma=0.15, \rho=0.01, N=50$ for GMFBM, and $N=2$ for MFBM.

Regarding the effect of the number of FBMs involved in the GMFBM, we calculate four sets of CDS prices as a function of $T$, under four different $N$ values, as shown in Fig 5. Note that the BM and MFBM models are special cases of the GMFBM model, and are corresponding to $N=1$ and $N=2$, respectively. From this figure, it is clear that the prices are approaching to the one under the BM once the number of the FBMs involved in the GMFBM increases. This implies that the currently adopted model can not only exhibit the long-range dependence, but also produce closer results to the BM model than the MFBM model.

\section{Conclusion}

In this paper, the pricing of the CDS is investigated under a GMFBM, which includes all the FBM models used in the literature as its special cases. After a sufficient condition for the GMFBM market to be arbitrage free is successfully established, a closed-form analytical expression for the price of the CDS is then derived based on the 


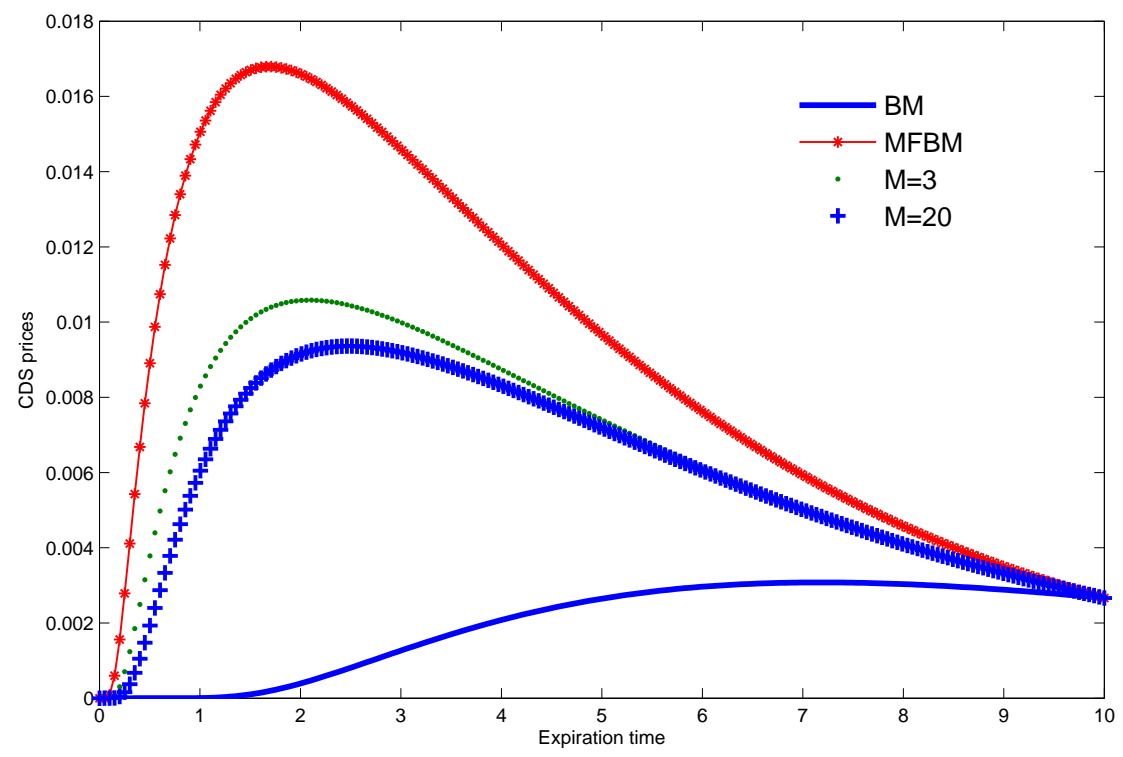

Figure 5. Variation of CDS prices with N. Model parameters are $V_{0}=100, r=0.06, \sigma=0.15, \rho=0.01, N=50$ for GMFBM, and $N=2$ for MFBM.

risk-neutral assumption. Finally, through numerical experiments, the quantitative impacts of different parameters on the price of the CDS are discussed.

\section{References}

[1] F. Biagini, Y.Z. Hu, B. $\phi$ ksendal, T.S. Zhang. Stochastic claculus for fractional Brownian motion and applications. Springer. (2008).

[2] T. Björk, H. Hult. A note on Wick products and the fractional Black-Scholes model. Finance and Stochastics 9(2005), 197-209.

[3] D. Brigo, K. Chourdakis. Counterparty risk for credit default swaps impact of spread volatility and default correlation. International Journal of Theoretical and Applied Finance (2013), to appear.

[4] P. Cheridito. Mixed fractional Brownian motion. Bernoulli 7(6)(2001), 913-934.

[5] P. Cheridito. Arbitrage in fractional Brownian motion models. Finance and Stochastics 7(2002), 533-553.

[6] E. de Malherbe. A simple probabilistic approach to the pricing of credit swap covenants. Journal of Risk 8(3)(2006), 85-113.

[7] D. Duffie, K.J. Singleton. An econometric model of the term structure of interest-rate swap yields. Journal of Finance 52(4)(1997), 1287-1321.

[8] T. Duncan, Y. Hu, B. Pasik-Duncan. Stochastic calculus for fractional Brownian motion, I. Theory. SIAM Journal on Control and Optimization 38(2000), 582-612.

[9] R.J. Elliott, J. van der Hoek. A general white nose theory and applications to finance. Mathematical Finance 13(2003), 301-330.

[10] X.J. He. Fractional models of credit risk pricing and related issues. In preparation (2013).

[11] D.A. Hsieth. Chaos and nonlinear dynamics: Applications to financial market. Journal of Finance 46(5)(1991), $1839-1877$.

[12] R. Jarrow, S. Turnbull. Pricing derivatives on financial securities subject to credit risk. Journal of Finance 50(1) (1995), 53-86.

[13] S.J. Lin. Stochastic analysis of fractional Brownian motion. Stochastics and Stochastic Reports 55(1995), 121-140.

[14] F.A. Longstaff, E.S. Schwartz. Valuing credit derivatives. Journal of Fixed Income 5(1)(1995), 6-12.

[15] D. Madan, H. Unal. Pricing the risks of default. Review of Derivatives Research 2(2)(1998), 121-160.

[16] B.B. Mandelbrot. When Can Price be Arbitraged Efficiently? A Limit to the Validity of the Random Walk and Martingale Models. The Review of Economics and Statistics 53(3)(1971), 225-236.

[17] B.B. Mandelbrot, M. S. Taqqu. Robust R/S analysis of long-run serial correlation. Proceedings of the 42nd session of the International Statistical Institut 48(1979), 69-99.

[18] M.C. Mariani, I. Florescu, M.P. Beccar Varela, E. Ncheuguim. Long corrections and Levy model applied to the study of memory effects in high frequency data. Physica A: Statistical Mechanics and its Applications 388(8)(2009), 1659-1664.

[19] R.C. Merton. On the pricing of corporate debt: the risk structure of interest rate. Journal of Finance 29(2)(1974), 449-470.

[20] D. O'Kane, S. Turnbull. Valuation of credit default swaps. Fixed Income Quantitative Credit Research Q1/Q3(2003), 1-17. 
[21] C. Rama. Long range dependence in financial market. Fractals in Engineering. Springer London. (2005), 158-179.

[22] J.A. Ramirez, J. Alvarrez, E. Rodriguez, G.F. Anaya. Time-varying Hurst exponent for US stock markets. Physica A: Statistical Mechanics and its Applications 387(24)(2008), 6159-6169.

[23] D. Shimko, N. Tejima and D. Van Deventer. The pricing of risky debt when interest rates are stochastics. Journal of Fixed Income 3(2)(1993), 437-444.

[24] X. Su, W. Wang. Pricing options with credit risk in a reduced form model. Journal of the Korean Statistical Society 41(4)(1993), 58-65.

[25] L. Sun. Pricing currency options in the mixed fractional Brownian motion. Physica A: Statistical Mechanics and its Applications 392(16)(2013), 3441-3458.

[26] X. Sun, L. Yan. Mixed-fractional models to credit risk pricing. Journal of Statistical and Econometric Methods 1(3)(2012), 79-96.

[27] H.P. Suryawan. Generalized mixed fractional Brownian motion as a generalized white noise functional. Working paper. Available at: http://binus.ac.id/wp-content/uploans/2012/02/02_Herry_setting.pdf (2012).

[28] C. Thäle. Further remarks on mixed fractional Brownian motion. Applied Mathematical Sciences 3(38)(2009), $1885-1901$.

[29] W.L. Xiao, W.G. Zhang and X.L. Zhang. Pricing model for equity warrants in a mixed fractional Brownian environment and its algorithm. Physica A: Statistical Mechanics and its Applications 391(24)(2012), 6418-6431.

[30] H. van Zanten. When is a linear combination of independent FBM's equivalent to a single FBM?. Stochastic Processes and their Applications 117(2007), 57-70

[31] W. Willinger, M.S. Taqqu, and V. Teverovsky. Stock market prices and long-range dependence. Finance and Stochastics 3(1)(1999), 1-13. 